\section{OPTIMIZACIÓN DEL MÉTODO DE DIGESTIÓN ÁCIDA EN SISTEMA DE MICROONDAS PARA LA DETERMINACIÓN DE CONCENTRACIONES PSEUDO- TOTALES DE Fe, Mn Y Zn EN SEDIMENTOS DE LA CUENCA KATARI BAJO, LA PAZ, BOLIVIA}

Full original article
Received 08052021

Accepted 12182021

Published 12302021

Vol. 38, No.5, pp. 194-208, Nov./Dec.2021

Revista Boliviana de Química

\begin{abstract}
38(5), 194-208, Nov./Dec. 2021
Bolivian Journal of Chemistry

DOI: $10.34098 / 2078-3949.38 \cdot 5.2$
\end{abstract}

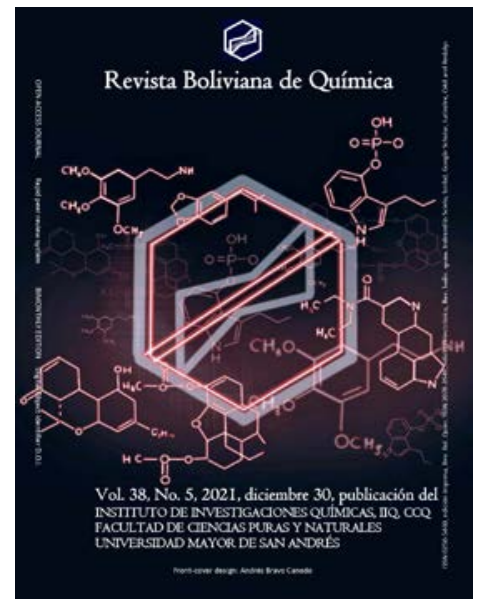

Delia M. Cori Condori, María I. Chambi Tapia, Leonardo Gúzman Alegria, Jorge Quintanilla Aguirre, Oswaldo E. Ramos Ramos*

Chemical Sciences Department, Instituto de Investigaciones Químicas IIQ, School of Pure and Natural Sciences FCPN, Universidad Mayor de San Andrés UMSA, P.O. Box 303, Calle Andrés Bello s/n Edificio IIQ, Campus Cota Cota, Phone +59122795878, La Paz, Bolivia, http://cienciasquimicas.umsa.bo/

Keywords: Trace Elements, Microwave System, Acid digestion, Sediments

Palabras clave: Elementos Traza, Microondas, Digestión ácida, Sedimentos

\title{
ABSTRACT
}

The sediments are considered reservoirs of trace elements where these are transported by river water and deposited in the lower part of the basin. The work presents the optimization of an acid digestion method in a microwave system to determine trace elements like Fe, Mn and $\mathrm{Zn}$ in sediments at three depths, PK9-1 (0-0.5 m), PK9-2 (0.5-2 m) and PK9-3 (2-2.7 m) in a randomly selected piezometer from a total of 17 piezometers. In addition, certified reference materials as the MRC MESS-3 for sediments and the MRC C17-2 for soils have been used for a quality control of the acid digestion method. For the quantification, the Flame Atomic Absorption Spectrometry (FAAS) technique has been used and for the statistical interpretation of the data, a 2x2 factorial design (ANOVA) was applied. The extraction procedures involve volume variations of $3: 3$ and $3: 1 \mathrm{~mL}$ of $\mathrm{HNO}_{3}: \mathrm{HCl}$, a power of 300/600 W and 100/300/700 W and with/without the use of $\mathrm{H}_{2} \mathrm{O}_{2}$. The result of the MRC MESS-3 shows that varying the extraction conditions does not considerably affect the concentrations of $\mathrm{Fe}, \mathrm{Mn}$ and $\mathrm{Zn}$, where the most optimal procedure is 3 hours, without $\mathrm{H}_{2} \mathrm{O}_{2}$ and with $3: 1 \mathrm{~mL} \mathrm{HNO}_{3} / \mathrm{HCl}$ with a minimum significant difference between the experimental and the reference values. The results using the MRC C17-2 show errors that exceed $10 \%$ where there are significant differences between the experimental and reference value for $\mathrm{Zn}$ and $\mathrm{Mn}$. On the other hand, PK9-1 (0-0.5) $\mathrm{m}$ and PK9-3 (0.5-2) m show $\mathrm{P}>0.05$ for Fe, indicating a significant difference in the average concentrations, and $\mathrm{P}<0.05$ for $\mathrm{Zn} / \mathrm{Mn}$ indicating that there are no significant differences in the average concentrations when varying the volume and power conditions. Likewise, PK9-2 (0.5-2) m shows $\mathrm{P}>0.05$ for $\mathrm{Zn} / \mathrm{Fe}$, indicating a significant difference in the 
average concentrations when varying the conditions of volume, power and with/without the use of $\mathrm{H}_{2} \mathrm{O}_{2}$. For Mg, a $\mathrm{P}<0.05$ indicates that there is no significant difference in the average concentrations. Therefore, varying amounts of volume, power and $\mathrm{H}_{2} \mathrm{O}_{2}$ do not considerably alter the trace elements concentrations and therefore the trace elements concentrations vary with depth according to the lithological characteristics of the sediments.

\section{*Correspondent autor: oramos@fcpn.edu.bo}

\section{RESUMEN}

Los sedimentos son considerados reservorios de elementos traza donde, son transportados por agua de ríos y depositados en la parte de baja de las cuencas. El trabajo presenta la optimización del un método de digestión ácida en un sistema de microondas para determinar elementos traza de Fe, Mn y Zn en sedimentos a profundidades de PK9-1 (0-0,5) m, PK9-2 (0,5-2) m y PK9-3 (2-2,7) m en un piezómetro seleccionado al azar de un total de 17 piezómetros. Además, se han utilizado los materiales de referencia certificado MRC MESS-3 de sedimentos y MRC C17-2 de suelos para un control de calidad del método de digestión acida. Para la cuantificación se ha empleado la técnica de Espectrometría de Absorción Atómica a la flama (FAAS) y para la interpretación estadística de los datos se aplicó un diseño factorial 2x2 (ANOVA). Los procedimientos de extracción involucran variaciones de volumen 3:3 y 3:1 mL de $\mathrm{HNO}_{3}$ : $\mathrm{HCl}$, potencia 300/600 W y 100/300/700 W y con o sin la adición de $\mathrm{H}_{2} \mathrm{O}_{2}$. Los resultados del MRC MESS-3 muestran que al variar las condiciones de extracción, éstas no afectan considerablemente las

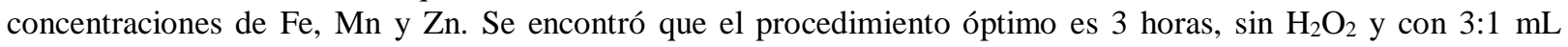
$\mathrm{HNO}_{3} / \mathrm{HCl}$ con una diferencia significativa mínima entre el valor experimental y el valor de referencia. Para un MRC C17-2 se tienen errores que superan el 10\% donde existen diferencias significativas entre el valor experimental y el valor de referencia para Zn y Mn. Por otro lado, el PK9-1 (0-0,5) m y PK9-3 (0,5-2) m muestran una P > 0,05 para Fe indicando diferencia significativa en las concentraciones promedio, y una $\mathrm{P}<0,05$ para $\mathrm{Zn} / \mathrm{Mn}$ indicando que no existe diferencias significativas en las concentraciones promedio al variar las condiciones de volumen y potencia. Así también PK9-2 (0,5-2) m muestra $\mathrm{P}>0,05$ para $\mathrm{Zn} / \mathrm{Fe}$ indicando una diferencia significativa en las concentraciones promedio al variar las condiciones de volumen, potencia y con o sin la adición de $\mathrm{H}_{2} \mathrm{O}_{2}$. Para Mn, la $\mathrm{P}<0,05$ indicando que no existe diferencia significativa en las concentraciones promedio. Por tanto, al variar cantidades de volumen, potencia y $\mathrm{H}_{2} \mathrm{O}_{2}$ no alteran considerablemente las concentraciones de los elementos traza y por ende las concentraciones de elementos traza varían con la profundidad de acuerdo a las características litológicas de los sedimentos.

\section{INTRODUCCIÓN}

Los sedimentos son una parte importante para el estudio de contaminación, más si es para el estudio de elementos traza, ya que es un medio de transporte en forma sólida que los libera a cuerpos de agua [1]. La movilización de los elementos traza están controlados fundamentalmente por condiciones de oxidación/reducción y pH. En los últimos años, varios estudios en sedimentos fueron realizados en Bolivia, entre ellos, la especiación de elementos traza a través de un método que caracteriza tres fracciones: 1) metales traza en forma disponible, 2) metales como reserva del sedimento, y 3) el contenido pseudo-total en elementos traza [2]. Otro estudio presenta la evaluación de un procedimiento de digestión de elementos traza realizado mediante la utilización de tres métodos de digestión: extracción por reflujo, calcinación en mufla y digestión ácida en horno de microondas [3]. También se realizó la evaluación de tres métodos de digestión ácida. Dos de estos métodos aplicables a sedimento, suelos y lodos y el tercer método aplicable para suelo [4]. Ormachea et al. presentaron un estudio de sedimentos explicando la geogénesis de los elementos traza y el As en sedimentos y aguas subterráneas [5]. En otro estudio se realizó el análisis de elementos traza en sedimentos de la Cuenca Katari Bajo de pozos de monitoreo utilizando el método de digestión ácida para determinar las concentraciones mediante la técnica de Fluorescencia de Rayos X por reflexión total [6]. Todos estos estudios usaron diferentes configuraciones y técnicas analíticas para la determinación de elementos traza pseudo-totales.

De acuerdo a estos antecedentes, se considera oportuno generar una metodología óptima para la cuantificación de los elementos traza (ET) que presente ventajas significativas. Por ejemplo, que sea aplicable a una diversidad de ET, y que el proceso de digestión por microondas disminuya los tiempos del ataque del ácido [11]. Debido a que la digestión por microondas se realiza en un sistema cerrado, ésta provoca un aumento en temperatura y presión de los recipientes. De esta forma, la energía entregada a la muestra y la agresividad del proceso es mayor [11], facilitando así, su destrucción pseudo-total. Por otro lado, las características litológicas y características fisicoquímicas como la

Downloadable from: Revista Boliviana de Química. http://www.bolivianchemistryjournal.org
195

http://www.scribd.com/bolivianjournalofchemistry 
materia orgánica (MO) formada a través de complejos, la salinidad (CE) sedimentada con sales de carbonatos, y pH determinaran las condiciones en las que los ET se encuentran en los sedimentos.

Por tanto, el presente trabajo presenta un análisis de la optimización del método de digestión ácida en un sistema cerrado de microondas para la determinación de concentraciones de elementos traza pseudo-total de Fe, Mn y Zn en sedimentos a tres diferentes profundidades de un piezómetro PK-9 ubicado en la parte baja de la Cuenca Katari Bajo del Altiplano Boliviano [7]. El seguimiento para el análisis se realizaró empleando dos materiales de referencias certificados: el MESS-3 (Marine Reference Materials for Trace Metals and other Constituents) material específico para sedimentos y el C17-2 (Certifícate of Analysis Metals in Soil) específico para suelos.

\section{EXPERIMENTAL}

\section{Área de estudio}

Las muestras de sedimentos fueron obtenidas por el proyecto de Polución y Biorremediación Acuática UMSA-ASDI (2015) de la Cuenca Katari Bajo ubicado en el departamento de La Paz (Bolivia). La zona está localizada entre las regiones de las poblaciones de Tambillo y la Bahía de Cohana (Figura 1). El acceso a la zona es mediante el tramo carretero El Alto - Laja Desaguadero y El Alto - Pucarani. El piezómetro (PK-9) fue elegido al azar de un total de 17 piezómetros y se encuentra localizado en la comunidad de Calería (Tabla 1). Las muestras de sedimentos fueron recolectadas a diferentes profundidades a medida que se realizaba la perforación de cada nivel de capa en bolsas ziplock. Seguidamente, las muestras fueron codificadas respecto a la profundidad y el lugar del cuál fueron tomadas para luego ser transportadas al laboratorio de Hidroquímica del Instituto de Investigaciones Químicas (IIQ) de la Universidad Mayor de San Andrés (UMSA) donde se realizaron los análisis de los parámetros fisicoquímicos.

Tabla 1. Características del piezómetro PK9

\begin{tabular}{cccccc}
\hline $\begin{array}{c}\text { Código } \\
\text { Piezómetro }\end{array}$ & Lugar & $\boldsymbol{X}$ & $\boldsymbol{Y}$ & $\begin{array}{c}\text { Nivel freático durante } \\
\text { la perforación }(\boldsymbol{m})\end{array}$ & $\begin{array}{c}\text { Prof. Perforada } \\
(\boldsymbol{m})\end{array}$ \\
\hline $\boldsymbol{P K 9}$ & Calería & 548284 & 8178288 & 1,9 & 4 \\
\hline
\end{tabular}

\section{MÉTODOS}

La metodología aplicada consiste en homogenizar la muestra de sedimento para el análisis en laboratorio. Para esto, se debe obtener una fracción fina de sedimento de $2 \mathrm{~mm}$ y $63 \mu \mathrm{m}$, que esté secada a temperatura ambiente para manipular y procesar. Los parámetros de $\mathrm{pH}, \mathrm{CE}, \mathrm{MO}$ y textura fueron analizados siguiendo procedimientos estandarizados por la Unidad de Análisis de Calidad Ambiental (UACA) perteneciente al IBTEN.

\section{Materiales y métodos}

\section{Parámetros fisicoquímicos}

Una característica litológica de los sedimentos expresa las proporciones relativas de las distintas fracciones de acuerdo con el tamaño de las partículas para la muestra. El piezómetro estudiado tiene tres profundidades: la primera PK9-1 entre 0 - 0,5 m que tiene una textura de arena, la segunda PK9-2 entre 0,5 - 2,0 m presenta una textura de franco-arcilloso-arenoso y la tercera PK9-3 entre 2,0 - 2,7 m con una textura de arena [8] (Figura 2).

El contenido de carbón orgánico (CO) de los sedimentos fue determinado por el método de Walkley y Black donde, la oxidación con dicromato de potasio $\left(\mathrm{K}_{2} \mathrm{Cr}_{2} \mathrm{O}_{7}\right)$ y la materia orgánica del sedimento (MO) fue calculada multiplicando el porcentaje de (CO) por el factor de 2 [11]. 


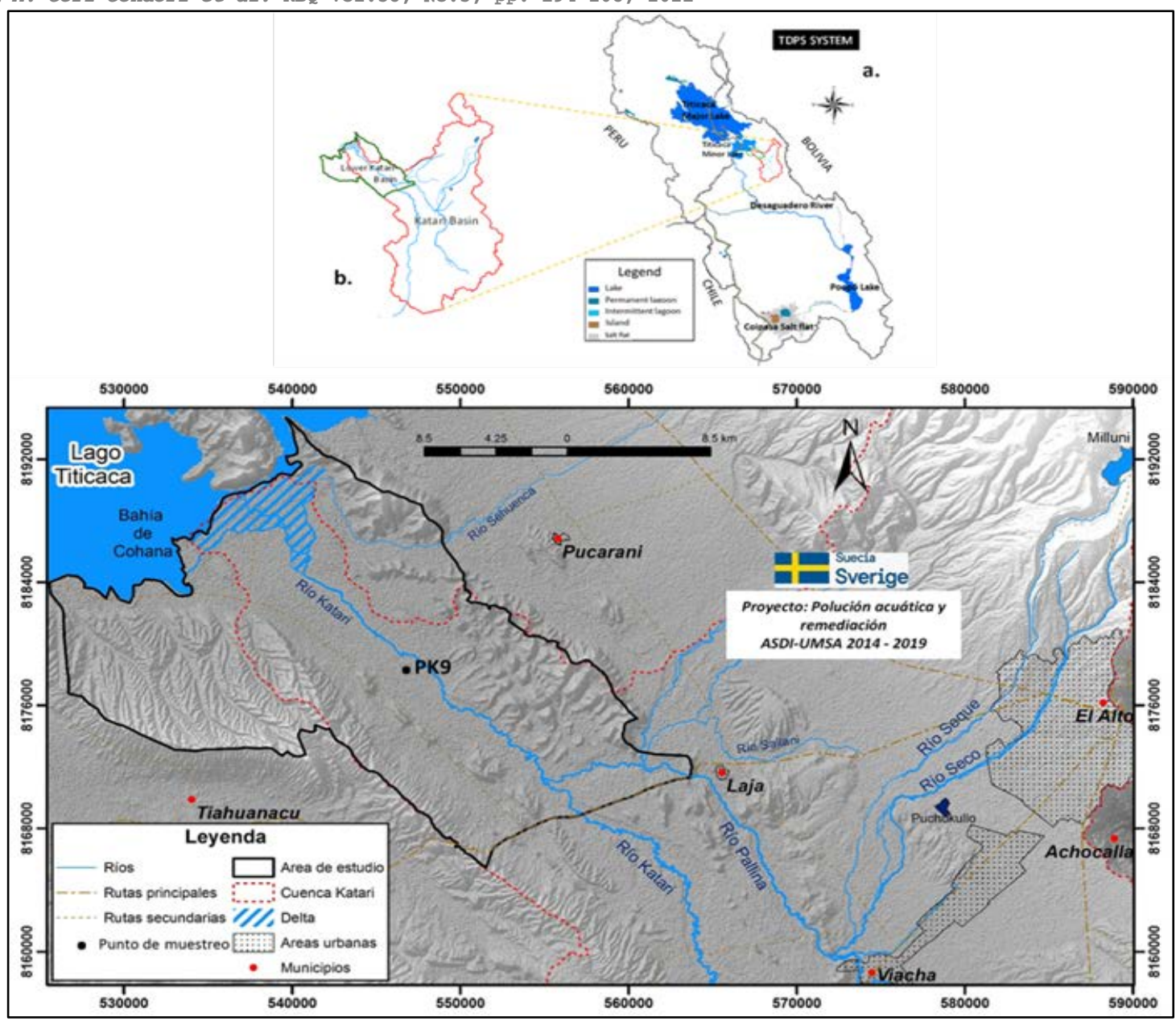

Figura 1. Área de estudio Cuenca Katari Bajo elaborado por PhD. Israel Quino

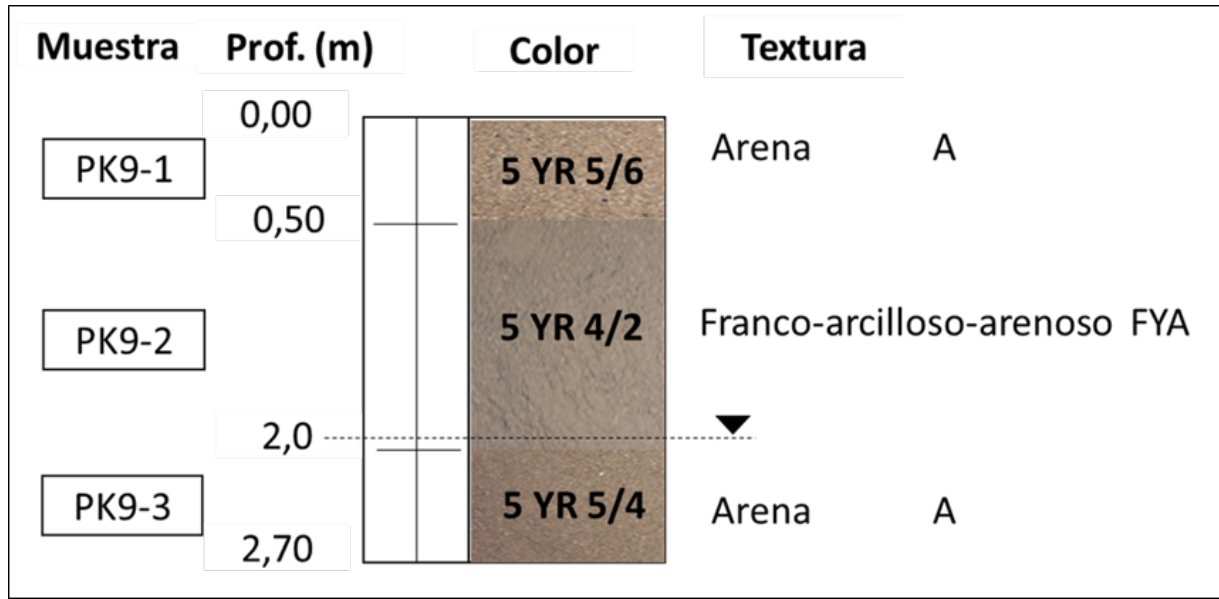

Figura 2. Perfil de sedimento del pozo de monitoreo (piezómetro PK-9) seleccionado al azar

\section{Digestión ácida y determinación de Fe, Mn y Zn}

Se han definido dos procedimientos de optimización para el tratamiento de los materiales de referencia MRC MESS3, C17-2 y muestras donde, se pesaron 0,1 g de MRC y 0,2 g de muestras PK9 en una balanza analítica $\pm 0,001 \mathrm{~g}$. Los procedimientos consisten en variar volúmenes de agua regia $\mathrm{HNO}_{3} / \mathrm{HCl}$, sin/con $\mathrm{H}_{2} \mathrm{O}_{2}$ al $30 \%$ y potencias de 300/600 W y 100/300/700 W (Tabla 3). 
Delia M. Cori Condori et al. RBQ Vol.38, No.5, pp. 194-208, 2021

Para descomponer los MRCs y muestras en un sistema cerrado, se ha empleado un equipo de microondas MICROWAVE 3000, ANTON PAAR. Cabe destacar que se ha establecido parámetros de presión de 75 bar, tiempo por rampa de 5 y 10 min, tiempo de mantenimiento por rampa de 5 y 10 min y ventilación (Figura 3).

La optimización del método fue realizada empleando blancos (matriz sin analito), cuatro replicas por MRC y muestras donde, se evaluaron la varianza para representar la variabilidad de los datos obtenidos, exactitud en términos del sesgo y el grado de concordancia entre el valor experimental y reporte de la hoja de MRC. Así también, se ha determinado el error sistemático aplicando la t-student al 95\% de confianza con grados de libertad de (gl= n-1) (Tabla 2).

Finalmente, el análisis elemental se realizó a través de la técnica de Espectrometría de Absorción Atómica a la Flama FAAS (PERKIN ELMER, ANALYST 200), para determinar la concentración pseudo-total de los analitos presente en las muestras de sedimentos.

Tabla 2. Parámetros estadísticos de medida

\begin{tabular}{ccc}
\hline Nombre & Formula & Nro. Ecuación \\
\hline Media & $\bar{x}=\frac{\sum_{i=1}^{n} x_{i}}{n}$ & Ecuación - 1 \\
\hline $\begin{array}{c}\text { Desviación } \\
\text { Estándar }\end{array}$ & $S=\sqrt{\frac{\sum_{i=1}^{n}\left(x_{i}-\bar{x}\right)^{2}}{n-1}}$ & Ecuación - 2 \\
\hline $\begin{array}{c}\text { Coeficiente de } \\
\text { variación }\end{array}$ & $\% C V=\frac{S}{\bar{X}} \times 100$ & Ecuación - 3 \\
\hline Varianza & $S^{2}=\frac{\sum_{i=1}^{n}\left(x_{i}-\bar{x}\right)^{2}}{n-1}$ & Ecuación - 4 \\
\hline $\begin{array}{c}\text { Exactitud } \\
\text { Sesgo }\end{array}$ & $S=\bar{x}-x_{a}$ & Ecuación - 5 \\
\hline Error & $t_{c a l}=\frac{\left[x_{a}-\bar{x}\right]}{S \times \sqrt{n}}$ & Ecuación - 6 \\
\hline & $\bar{x} \pm t \times \frac{D S}{\sqrt{n}}$ & Ecuación - 7 \\
\hline
\end{tabular}

Tabla 3. Procedimientos de digestión ácida en el horno de microondas a las diferentes potencias W

\begin{tabular}{lccccc}
\hline & Rampas (W) & $\begin{array}{c}\text { Presión } \\
\text { (bar) }\end{array}$ & $\mathbf{H N O}_{3}(\mathbf{m L})$ & $\mathbf{H C l}(\mathbf{m L})$ & $\mathbf{H}_{2} \mathbf{O}_{2}(\mathbf{m L})$ \\
\cline { 2 - 6 } PROCEDIMIENTO 1 & 300 & 75 & 3 & 3 & 1 \\
& 600 & & 3 & 1 & 1 \\
\hline \multirow{2}{*}{ PROCEDIMIENTO 2 } & 100 & 75 & 3 & 3 & 1 \\
& 300 & & 3 & 1 & 1 \\
\hline
\end{tabular}
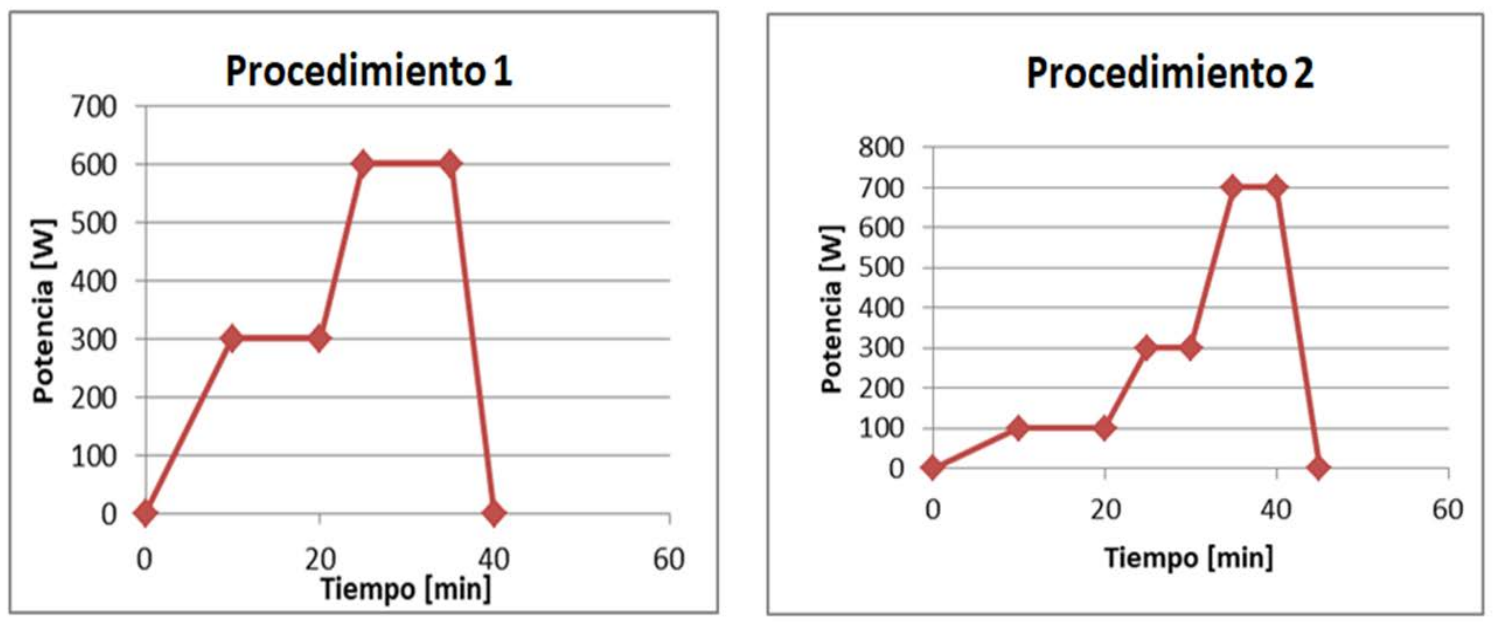

Figura 3. Esquema del procedimiento para la digestión ácida: a) 300/600 W de potencia; b) 100/300/700 W de potencia 


\section{RESULTADOS Y DISCUSIONES}

\section{Resultados de parámetros fisicoquímicos}

Los valores de pH para las tres profundidades se encuentran en un rango de 7,3 a 8,6 clasificados como muy débil a alta alcalinidad. La conductividad eléctrica (CE) se encuentra en un rango de $50 \mu \mathrm{S} / \mathrm{cm}$ en la parte superficial, 217 $\mu \mathrm{S} / \mathrm{cm}$ parte media y $101 \mu \mathrm{S} / \mathrm{cm}$ parte profunda indicando clasificación salino-alcalino [11]. El contenido de materia orgánica (\%MO) varia en un rango de 0,273 \% parte superficial, 3,460 \% parte media y 0,542 \% parte profunda con mayor contenido de materia orgánica en la segunda profundidad [9]. Este mayor contenido de MO podría disminuir la disponibilidad de los metales por complejación debido a la formación de complejos metálicos y sulfuros metálicos insolubles que son sumideros importantes para elementos traza en los sedimentos [10].

\section{Resultados de las concentraciones de los materiales de referencia MRC}

La optimización de la digestión ácida para la cuantificación de elementos traza fue realizada empleando dos materiales de referencia: MESS-3 específico para sedimentos y C17-2 específico para suelos.

La tabla 4 resume los resultados de las concentraciones de Fe, Mn y Zn con el MRC (MESS-3) con tratamiento de potencia de 300W/600W para encontrar las condiciones óptimas. Las condiciones óptimas fueron las siguientes: MESS-3-1 con un tiempo de digestión de 3 horas, con $\mathrm{H}_{2} \mathrm{O}_{2}$ y con 3:3 mL $\mathrm{HNO}_{3} / \mathrm{HCl}$; MESS-3-2 con un tiempo de 3 horas, sin $\mathrm{H}_{2} \mathrm{O}_{2}$ y con 3:3 mL $\mathrm{HNO}_{3} / \mathrm{HCl}$; MESS-3-3 con un tiempo de 15 minutos, sin $\mathrm{H}_{2} \mathrm{O}_{2}$ y con 3:1 mL $\mathrm{HNO}_{3} / \mathrm{HCl}$; y MESS-3-4 con un tiempo de 3 horas, sin $\mathrm{H}_{2} \mathrm{O}_{2}$ y con 3:1 mL $\mathrm{HNO}_{3} / \mathrm{HCl}$. En todos los casos, las concentraciones de los ET muestran errores aceptables. Por tanto, al variar las condiciones de tiempo de predigestión, con $\mathrm{H}_{2} \mathrm{O}_{2}$ y $\mathrm{HNO}_{3} / \mathrm{HCl}$ no afecta considerablemente las concentraciones de ET presentes en el MRC.

La tabla 6 resume los resultados de las concentraciones de Zn y Mn en el MRC C17 para el experimento C172-1. Para éste, las condiciones óptimas del método fueron con tratamiento de potencia de 300W/600W, tiempo de 3 horas, con $\mathrm{H}_{2} \mathrm{O}_{2}$ y 3:1 mL $\mathrm{HNO}_{3} / \mathrm{HCl}$. Para el experimento C17-2-2, las condiciones óptimas fueron con un tiempo de 15 minutos, sin $\mathrm{H}_{2} \mathrm{O}_{2}$ y con 3:1 mL $\mathrm{HNO}_{3} / \mathrm{HCl}$. Los resultados muestran que, variar las condiciones de tiempo de pre-digestión, como las cantidades de $\mathrm{H}_{2} \mathrm{O}_{2}$ y $\mathrm{HNO}_{3} / \mathrm{HCl}$, no afecta considerablemente las concentraciones de $\mathrm{Zn}$. La tabla 8 resume los resultados de C17-2 con tratamiento de potencia de 100W/300W/700W, con un tiempo de 3 horas, con $\mathrm{H}_{2} \mathrm{O}_{2}$ y 3:3 mL $\mathrm{HNO}_{3} / \mathrm{HCl}$; y C17-2-4 con un tiempo de 3 horas, sin $\mathrm{H}_{2} \mathrm{O}_{2}$ y con 3:3 mL $\mathrm{HNO}_{3} / \mathrm{HCl}$ y C17-2-5 con un tiempo de 3 horas, con $\mathrm{H}_{2} \mathrm{O}_{2}$ y con 3:1 mL $\mathrm{HNO}_{3} / \mathrm{HCl}$ donde, al variar las condiciones afectan considerablemente las concentraciones de $\mathrm{Mn}$.

Tabla 4. Resultados de las concentraciones pseudo-totales de ET en MRC MESS-3

\begin{tabular}{|c|c|c|c|c|c|}
\hline \multicolumn{6}{|c|}{ Potencia 300/600 W } \\
\hline Analito & $\#$ & Código & mg/kg & $\begin{array}{l}\text { MRC } \\
\mathrm{mg} / \mathrm{kg}\end{array}$ & $\begin{array}{c}\text { Error } \\
\%\end{array}$ \\
\hline \multirow{4}{*}{ Zn } & 1 & MESS-3-1 & 153,386 & \multirow{4}{*}{$159 \pm 8$} & 3,531 \\
\hline & 2 & MESS-3-2 & 163,376 & & 2,752 \\
\hline & 3 & MESS-3-3 & 155,258 & & 2,353 \\
\hline & 4 & MESS-3-4 & 157,480 & & 0,956 \\
\hline \multirow{4}{*}{ Mn } & 1 & MESS-3-1 & 356,574 & \multirow{4}{*}{$324 \pm 12$} & 10,054 \\
\hline & 2 & MESS-3-2 & 353,406 & & 9,076 \\
\hline & 3 & MESS-3-3 & 367,063 & & 13,291 \\
\hline & 4 & MESS-3-4 & 372,539 & & 14,981 \\
\hline \multirow{5}{*}{$\mathbf{F e}$} & Nro. & Código & $\% \mathrm{~m}$ & \%MRC & \%Error \\
\hline & 1 & MESS-3-1 & 4,029 & \multirow{4}{*}{$4,34 \pm 0,11$} & 7,169 \\
\hline & 2 & MESS-3-2 & 4,023 & & 7,311 \\
\hline & 3 & MESS-3-3 & 3,889 & & 10,394 \\
\hline & 4 & MESS-3-4 & 3,986 & & 8,151 \\
\hline
\end{tabular}

Downloadable from: Revista Boliviana de Química. http://www.bolivianchemistryjournal.org
199

Volumen $38 \mathrm{~N}^{\circ} 5$. Año 2021

http://www.scribd.com/bolivianjournalofchemistry 
Tabla 5. Resultados de los parámetros estadísticos de ET en MRC MESS-3 con potencia 300/600 W

\begin{tabular}{ccccccccc}
\hline & \multicolumn{7}{c}{ MESS-3 } \\
\hline Elementos & Media & ES & DS & \%CV & $\boldsymbol{S}^{2}$ & Sesgo & t cal & t crit \\
Zn & 157,4 & \pm 7 & 4,3 & 2,8 & 18,8 & 1,6 & 0,187 & 3,182 \\
Mn & 362,4 & \pm 14 & 8,9 & 2,5 & 79,8 & 38,4 & 2,149 & 3,182 \\
Fe & 4,0 & $\pm 0,10$ & 0,1 & 1,6 & 0,004 & 0,4 & 2,771 & 3,182 \\
\hline \multicolumn{7}{c}{ Criterio de aceptación } \\
\hline \multicolumn{7}{c}{ t cal $<$ t crit } \\
\hline \multicolumn{10}{c}{ Exactitud: Zn, Mn, Fe }
\end{tabular}

DS = Desviación Estándar; ES = Error Sistemático; CV= Coeficiente de Variación; $\boldsymbol{S}^{2}=$ Varianza

Tabla 6. Resultados de la concentración pseudo-total de ET en MRC C17-2

\begin{tabular}{cccccc}
\hline \multicolumn{7}{c}{ Nro. } & Código & Potencia 300/600 W & & \\
\hline & & & Mg/g & $\boldsymbol{\mu g} / \mathbf{g}$ & $\begin{array}{c}\text { Error } \\
\mathbf{\%}\end{array}$ \\
\hline Zn & 1 & C17-2-1 & 1792,453 & $\mathbf{1 7 2 5} \pm \mathbf{3 1 7}$ & 3,910 \\
& 2 & C17-2-2 & 1726,191 & & 0,011 \\
\hline Mn & 1 & C17-2-1 & 1812,314 & $\mathbf{1 4 0 5} \pm \mathbf{2 0 7}$ & 28,990 \\
& 2 & C17-2-2 & 1780,754 & & 26,744 \\
\hline
\end{tabular}

Tabla 7. Resultados de los parámetros estadísticos de ET en MRC C17-2 con potencia 300/600 W

\begin{tabular}{|c|c|c|c|c|c|c|c|c|}
\hline & & & & C17-2 & & & & \\
\hline Elementos & Media & ES & DS & $\% \mathrm{CV}$ & S2 & Sesgo & t cal & t crit \\
\hline Zn & 1759,3 & \pm 421 & 46,9 & 2,7 & 2195,3 & 34,3 & 0,518 & 12,706 \\
\hline Mn & 1796,5 & \pm 201 & 22,3 & 1,2 & 498,0 & 391,5 & 12,406 & 12,706 \\
\hline \multicolumn{9}{|c|}{ criterio de aceptación } \\
\hline \multicolumn{3}{|c|}{$\begin{array}{r}\text { Exactitud: Zn } \\
\text { Mn }\end{array}$} & \multicolumn{3}{|c|}{$\begin{array}{l}\text { t cal }<\text { t crit } \\
t \text { cal } \leq t \text { crit }\end{array}$} & \multicolumn{3}{|c|}{$\begin{array}{c}\text { Cumple } \\
\text { No cumple }\end{array}$} \\
\hline
\end{tabular}

Tabla 8. Resultados de la concentración pseudo-total de ET para el MRC C17-2

\begin{tabular}{|c|c|c|c|c|c|}
\hline \multicolumn{6}{|c|}{ Potencia 100/300/700 W } \\
\hline & Nro. & Código & $\mathbf{m g} / \mathbf{k g}$ & $\begin{array}{l}\text { MRC } \\
\mathrm{mg} / \mathrm{kg}\end{array}$ & $\begin{array}{c}\text { Error } \\
\%\end{array}$ \\
\hline \multirow{3}{*}{ Zn } & 3 & C17-2-3 & 1780,754 & \multirow{3}{*}{$1725 \pm 317$} & 3,232 \\
\hline & 4 & C17-2-4 & 1805,556 & & 4,670 \\
\hline & 5 & C17-2-5 & 1777,888 & & 3,066 \\
\hline \multirow{3}{*}{ Mn } & 3 & C17-2-3 & 1790,675 & \multirow{3}{*}{$1405 \pm 207$} & 27,450 \\
\hline & 4 & C17-2-4 & 2113,095 & & 50,398 \\
\hline & 5 & C17-2-5 & 1802,789 & & 28,312 \\
\hline
\end{tabular}

Tabla 9. Resultados de los parámetros estadísticos de ET en MRC C17-2 con Potencia 100/300/700 W

\begin{tabular}{|c|c|c|c|c|c|c|c|c|}
\hline \multicolumn{9}{|c|}{ C17-2 } \\
\hline Elementos & Media & ES & DS & $\% \mathrm{CV}$ & S2 & Sesgo & t cal & t crit \\
\hline Zn & 1779,3 & \pm 38 & 2,0 & 0,1 & 4,1 & 54,321 & 18,957 & 12,706 \\
\hline Mn & 1796,7 & \pm 454 & 8,6 & 0,5 & 73,4 & 391,7 & 32,336 & 12,706 \\
\hline \multicolumn{9}{|c|}{ Criterio de aceptación } \\
\hline \multicolumn{3}{|c|}{$\begin{aligned} & \text { Exactitud: } \mathbf{Z n}, \mathbf{M n} \\
\text { DS = } & \text { Desviación Estándar; ES = Err }\end{aligned}$} & \multicolumn{3}{|c|}{ t cal > t crit } & \multicolumn{3}{|c|}{ No cumple } \\
\hline
\end{tabular}


Los resultados estadísticos para un MRC MESS-3 muestra criterios de aceptación óptima para Zn, Mn y Fe con tcal < tcrit, indicando que no existe diferencia significativa entre el valor experimental y el valor reportado en la hoja de MRC (Tabla 5). Por otro lado, los valores de $\% \mathrm{CV}<5 \%$ demuestra que no existe variabilidad en los datos obtenidos durante la digestión (Tabla 5). A partir de estos resultados se logró determinar el procedimiento óptimo con un tiempo de 3 horas, sin $\mathrm{H}_{2} \mathrm{O}_{2}$ y con 3:1 mL $\mathrm{HNO}_{3} / \mathrm{HCl}$. Así también, para el MRC C17-2 con potencia de 300/600 W, muestra que tcal < tcrit para Zn donde, no existe diferencia significativa. En el caso de Mn, el tcal $\leq$ tcrit indicando que existe diferencia significativa entre los valores experimentales y valores de referencia y $\% \mathrm{CV}<5 \%$ con variabilidad mínima de los datos (Tabla 7). Los resultados para un MRC C17-2 con potencia de 100/300/700 W muestran tcal > tcrit para $\mathrm{Zn}$ y Mn, indicando diferencias significativas entre el valor experimental y el valor reportado en la hoja de MRC. Ésto demuestra que el procedimiento 2 no es óptimo para determinar dichos ET (Tabla 9). Por tanto, el procedimiento óptimo es a potencia de 300/600 W con un tiempo de 15 minutos, $\sin \mathrm{H}_{2} \mathrm{O}_{2}$ y 3:1 mL $\mathrm{HNO}_{3} / \mathrm{HCl}$.

\section{Concentración pseudo-total de ET de una muestra PK-9 de sedimento: representados con un diseño factorial de $2 X 2$}

A través de un análisis estadístico de un diseño factorial 2x2 (ANOVA) con el programa SPSS en las muestras del piezómetro PK-9 se ha determinado la interacción que existe al variar factores como potencias de 300/600 W y 100/300/700 W, relación volumen 3:3 mL y 3:1 mL de $\mathrm{HNO}_{3} / \mathrm{HCl}$, y con/sin $\mathrm{H}_{2} \mathrm{O}_{2}$ al $30 \%$ en una muestra de sedimentos a profundidades de PK9-1 (0-0,5) m, PK9-2 (0,5-2,0) m y PK9-3 (2,0-2,70) m.

\section{Resultados de las concentraciones pseudo-totales de Zn, Mn y Fe de PK9-1 (0-0,5 m)}

La figura 4 resume las interacciones de las concentraciones pseudo-totales de Zn, Mn y Fe en sedimentos de la primera profundidad (PK9-1) con variación de factores de potencias de 300/600 W y 100/300/700 W, y relación de volumen 3:3 mL y 3:1 mL de $\mathrm{HNO}_{3} / \mathrm{HCl}$. La figura 4.1a) con interacciones rampa*volumen muestra la variabilidad en las concentraciones de $\mathrm{Zn}$, donde al variar los factores de potencia $300 / 600 \mathrm{~W}$ y $3: 3 \mathrm{~mL} \mathrm{HNO} / \mathrm{HCl}$ con respecto a potencia 300/600 W y 3:1 $\mathrm{mL} \mathrm{HNO}_{3} / \mathrm{HCl}$ no existe variación en la concentración de Zn. Por otro lado, para potencia 100/300/700 W y 3:3 mL $\mathrm{HNO}_{3} / \mathrm{HCl}$ se obtiene concentraciones bajas de $\mathrm{Zn}$ con respecto a potencia 100/300/700 W y 3:1 $\mathrm{mL} \mathrm{HNO}_{3} / \mathrm{HCl}$ donde se obtiene mayor concentración. La figura 4.2a) con interacciones volumen*rampa muestra la variabilidad en las concentraciones de $\mathrm{Zn}$, donde al variar el volumen $3: 3 \mathrm{~mL} \mathrm{HNO}_{3} / \mathrm{HCl}$ y potencia 100/300/700 W se obtiene concentraciones bajas con respecto a volumen 3:3 $\mathrm{mL} \mathrm{HNO}_{3} / \mathrm{HCl}$ y potencia 300/600 W. Así mismo, para un volumen de $3: 1 \mathrm{~mL} \mathrm{HNO}_{3} / \mathrm{HCl}$ y potencia $100 / 300 / 700 \mathrm{~W}$ se obtiene concentraciones mayores con respecto a un volumen de $3: 1 \mathrm{~mL} \mathrm{HNO}_{3} / \mathrm{HCl}$ y potencia 300/600 W.

La figura 4.1b) con interacciones rampa*volumen muestra la variabilidad en las concentraciones de $\mathrm{Mn}$, donde al variar los factores de potencia $300 / 600 \mathrm{~W}$ y $3: 3 \mathrm{~mL} \mathrm{HNO} / \mathrm{HCl}$ se obtiene bajas concentraciones con respecto a potencia 300/600 W y 3:1 mL $\mathrm{HNO}_{3} / \mathrm{HCl}$. Por otro lado, para potencia 100/300/700 W y 3:3 mL $\mathrm{HNO}_{3} / \mathrm{HCl}$ se obtiene concentraciones altas de $\mathrm{Mn}$ con respecto a potencia 100/300/700 W y 3:1 $\mathrm{mL} \mathrm{HNO} / \mathrm{HCl}$ donde se obtiene menor concentración de Mn. La figura 4.2b) con interacciones volumen*rampa muestra variabilidad en las concentraciones de $\mathrm{Mn}$, donde al variar el volumen $3: 3 \mathrm{~mL} \mathrm{HNO}_{3} / \mathrm{HCl}$ y potencia $300 / 600 \mathrm{~W}$ se obtiene concentraciones bajas con respecto a volumen $3: 3 \mathrm{~mL} \mathrm{HNO}_{3} / \mathrm{HCl}$ y potencia 100/300/700 W. Así, para un volumen de 3:1 $\mathrm{mL} \mathrm{HNO}_{3} / \mathrm{HCl}$ y potencia 300/600 W se obtiene concentraciones bajas con respecto a un volumen de 3:1 mL $\mathrm{HNO}_{3} / \mathrm{HCl}$ y potencia $100 / 300 / 700 \mathrm{~W}$ donde las diferencias en las concentraciones son significativas.

La figura 4.1c) con interacciones rampa*volumen muestra variabilidad en las concentraciones de Fe, donde al variar los factores de potencia 300/600 W y 3:1 $\mathrm{mL} \mathrm{HNO}_{3} / \mathrm{HCl}$ se obtiene muy bajas concentraciones con respecto a potencia 300/600 W y 3:3 mL $\mathrm{HNO}_{3} / \mathrm{HCl}$. Por otro lado, para potencia $100 / 300 / 700 \mathrm{~W}$ y $3: 1 \mathrm{~mL} \mathrm{HNO}_{3} / \mathrm{HCl}$ se obtiene concentraciones muy bajas de Fe con respecto a potencia 100/300/700 W y 3:3 mL HNO $3 / \mathrm{HCl}$.

La figura 4.2c) con interacciones volumen*rampa muestra la variabilidad en las concentraciones de $\mathrm{Fe}$, donde al variar el volumen $3: 3 \mathrm{~mL} \mathrm{HNO}_{3} / \mathrm{HCl}$ y potencia /100/300/700 W se obtiene concentraciones relativamente bajas con respecto a volumen $3: 3 \mathrm{~mL} \mathrm{HNO}_{3} / \mathrm{HCl}$ y potencia $300 / 600 \mathrm{~W}$. Además, para un volumen de $3: 1 \mathrm{~mL} \mathrm{HNO}_{3} / \mathrm{HCl}$ y potencia 300/600 W se obtiene concentraciones relativamente bajas con respecto a un volumen de 3:1 mL $\mathrm{HNO}_{3} / \mathrm{HCl}$ y potencia 100/300/700 W donde las diferencias en las concentraciones son significativas para ambos puntos de interacción.

Downloadable from: Revista Boliviana de Química. http://www.bolivianchemistryjournal.org
201

olumen 38 N5. Año 2021

http://www.scribd.com/bolivianjournalofchemistry 
ISSN 0250-5460 Rev. Bol. Quim. Paper edition

ISSN 2078-3949 Rev, boliv, Quim. Electronic edition

Delia M. Cori Condori et al. RBQ Vol.38, No.5, pp. 194-208, 2021
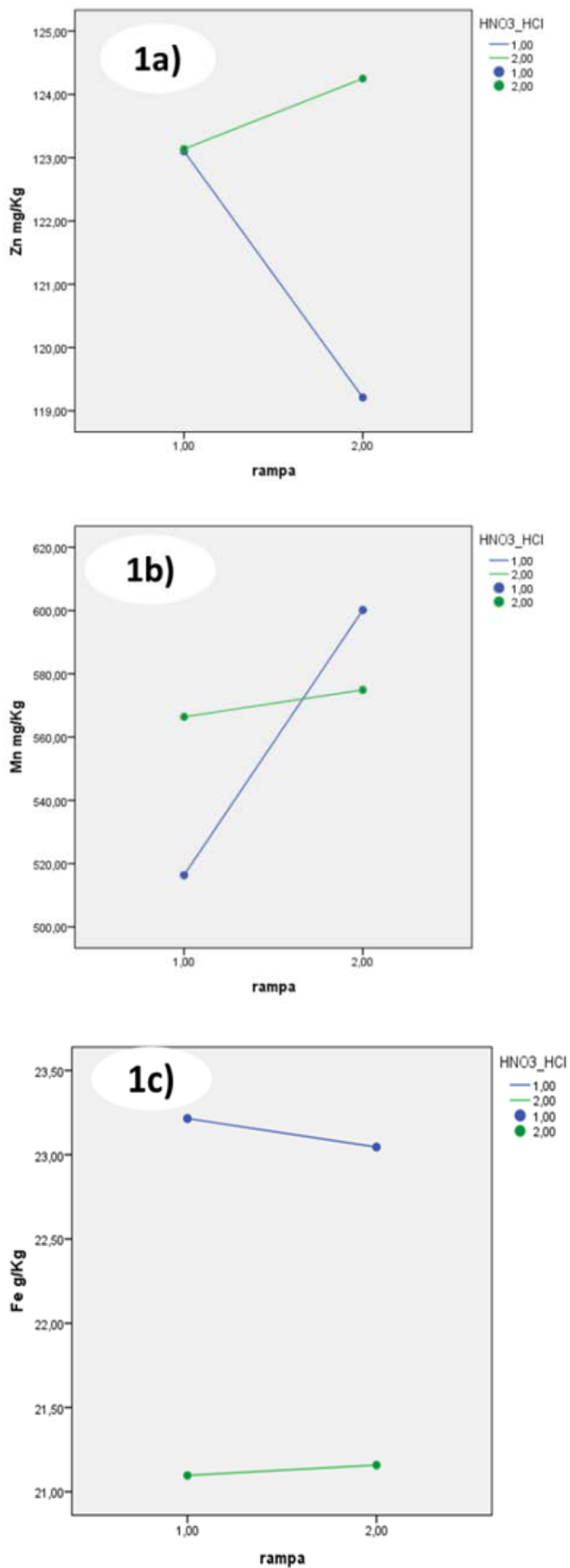
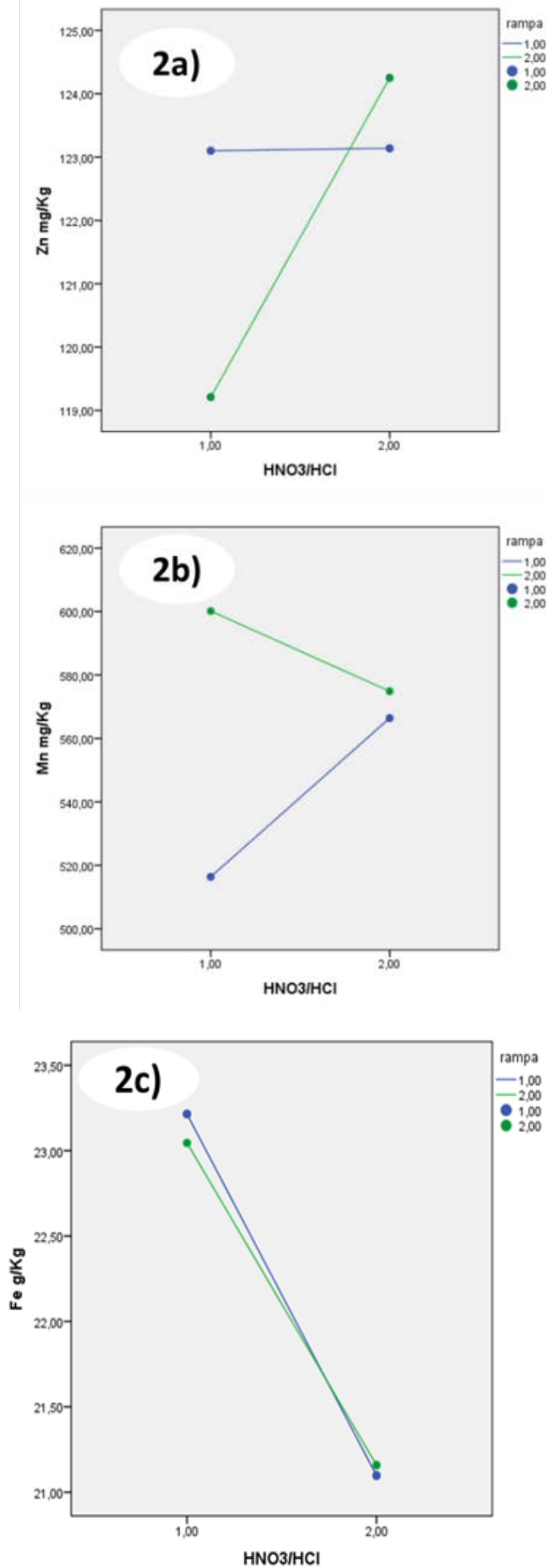

Figura 4. Diagrama factorial 2x2, 1a) y 2a) para el analito $\mathrm{Zn}, 1 b$ ) y 2b) para el Mn, 1c) y 2c) para el Fe variando la potencia (rampa 1; 300/600 W y rampa 2; 100/300/700 W) y volumen de ácidos (1; $\mathrm{HNO}_{3} / \mathrm{HCl} \mathrm{3:1} \mathrm{v/v} \mathrm{y} \mathrm{2;} \mathrm{HNO}_{3} / \mathrm{HCl} \mathrm{3:3} \mathrm{v/v)}$ 
Resultados de las concentraciones pseudo-totales de Zn, Mn y Fe de PK9-2 (0,5-2,0 m)
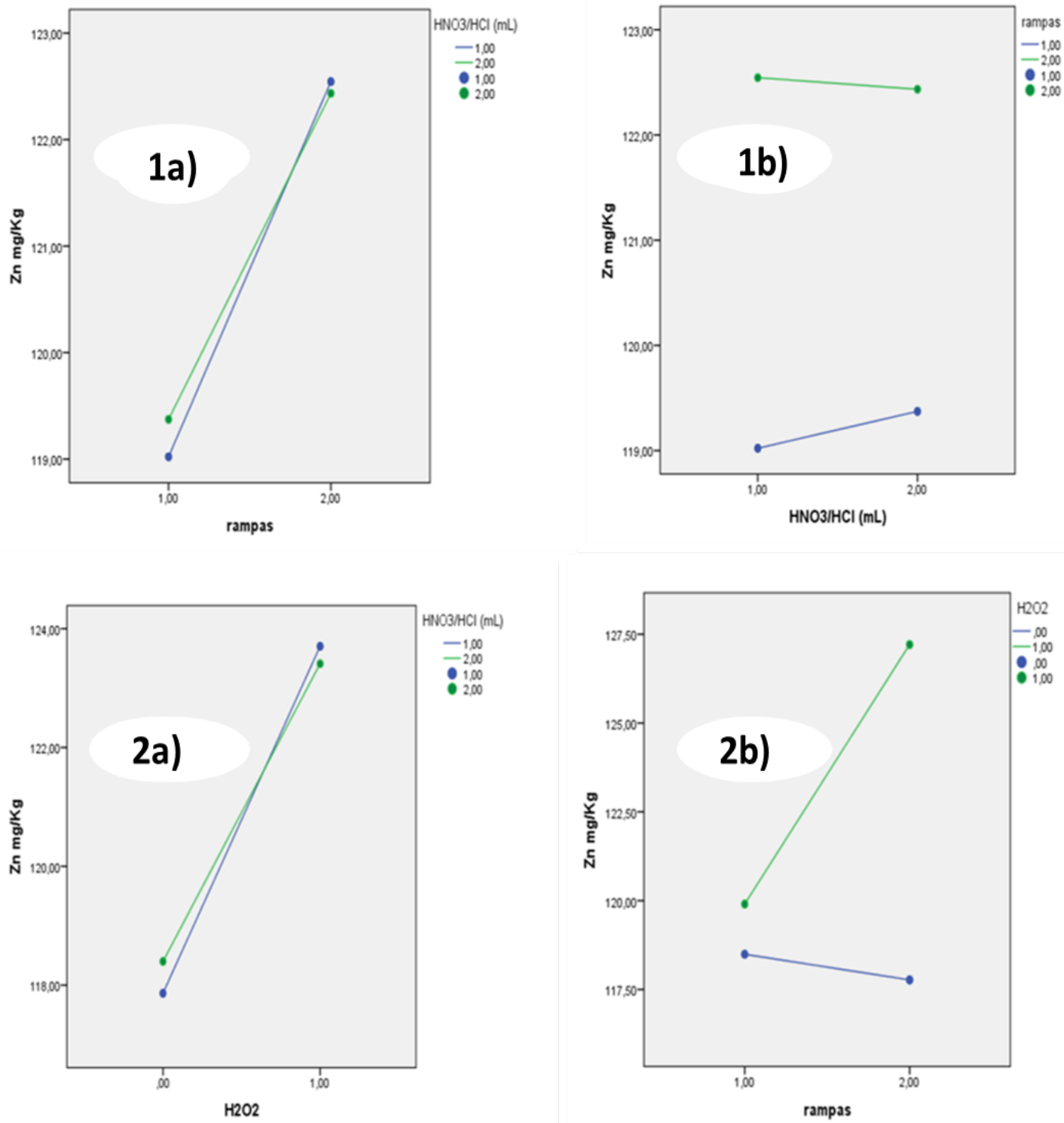

Figura 5. Diagrama factorial $2 x 2$ para el Zn variando potencia (rampa 1; 300/600 W y rampa 2; 100/300/700 W), volumen de ácido (1; $\mathrm{HNO}_{3}: \mathrm{HCl} \mathrm{3:1} \mathrm{v/v} \mathrm{y} \mathrm{2;} \mathrm{HNO}_{3}: \mathrm{HCl} \mathrm{3:3} \mathrm{v/v)} \mathrm{y} \mathrm{volumen} \mathrm{de} \mathrm{H}_{2} \mathrm{O}_{2}\left(1 ; 1 \mathrm{~mL} \mathrm{H}_{2} \mathrm{O}_{2} 2 ; 0 \mathrm{~mL} \mathrm{H}_{2} \mathrm{O}_{2}\right)$

La figura 5 resume las interacciones de las concentraciones pseudo-totales de Zn, Mn y Fe en sedimentos de la segunda profundidad PK9-2 con variación de factores de potencias de 300/600 W y 100/300/700 W, relación de volumen 3:3 y 3:1 mL $\mathrm{HNO}_{3} / \mathrm{HCl}$, y con/sin $\mathrm{H}_{2} \mathrm{O}_{2}$. La figura 5.1a) con interacciones rampa*volumen muestra variabilidad en las concentraciones de $\mathrm{Zn}$, donde al variar los factores de potencia 300/600 $\mathrm{W}$ y volumen 3:3 y 3:1 $\mathrm{mL} \mathrm{HNO}_{3} / \mathrm{HCl}$ se obtiene bajas concentraciones con respecto a potencia 100/300/700 W y volumen 3:3 mL y 3:1 $\mathrm{mL} \mathrm{HNO}_{3} / \mathrm{HCl}$ donde se obtiene mayores concentraciones de $\mathrm{Zn}$. La figura 5.1b) con interacciones volumen*rampa muestra variabilidad en las concentraciones de $\mathrm{Zn}$, donde al variar el volumen $3: 3 \mathrm{~mL} \mathrm{HNO} / \mathrm{HCl}$ y potencia 300/600 $\mathrm{W}$ se obtiene concentraciones muy bajas con respecto a volumen $3: 3 \mathrm{~mL} \mathrm{HNO} / \mathrm{HCl}$ y potencia $100 / 300 / 700 \mathrm{~W}$. Por otro lado, para un volumen de $3: 1 \mathrm{~mL} \mathrm{HNO}_{3} / \mathrm{HCl}$ y potencia 300/600 W se obtiene concentraciones muy bajas con respecto a un volumen de $3: 1 \mathrm{~mL} \mathrm{HNO}_{3} / \mathrm{HCl}$ y potencia 100/300/100 W. La figura 5.2a) muestra interacciones con/sin $\mathrm{H}_{2} \mathrm{O}_{2}$ *volumen con una diferencia significativa, donde al añadir $\mathrm{H}_{2} \mathrm{O}_{2}$ las concentraciones obtenidas de $\mathrm{Zn}$ son mayores con respecto $\sin \mathrm{H}_{2} \mathrm{O}_{2}$. La figura 5.2b) muestra la interacción rampas* con/sin $\mathrm{H}_{2} \mathrm{O}_{2}$, donde se muestra 
mayor concentración de $\mathrm{Zn}$ para potencia 100/300/700 W y con $\mathrm{H}_{2} \mathrm{O}_{2}$ y bajas concentraciones de $\mathrm{Zn}$ con potencias de $300 / 600 \mathrm{~W}$ y $\sin \mathrm{H}_{2} \mathrm{O}_{2}$.
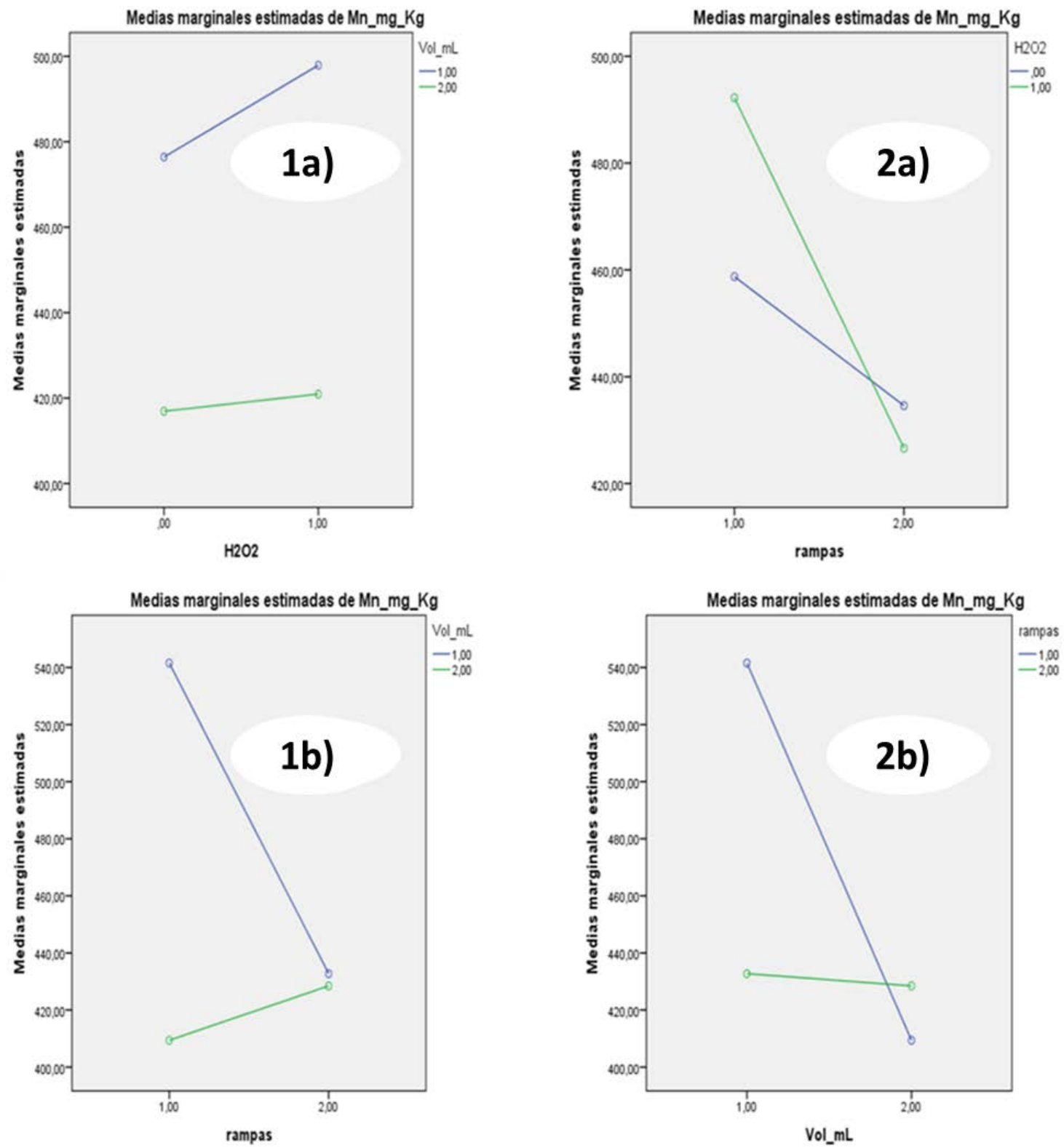

Figura 6. Diagrama factorial 2x2 para el Mn variando potencia (rampa 1; 300/600 W y rampa 2; 100/300/700 W), volumen de ácido $\left(1 ; \mathrm{HNO}_{3}: \mathrm{HCl} \mathrm{3:1} \mathrm{v/v} \mathrm{y} \mathrm{2;} \mathrm{HNO}_{3}: \mathrm{HCl} \mathrm{3:3} \mathrm{v/v)} \mathrm{y} \mathrm{volumen} \mathrm{de} \mathrm{H}_{2} \mathrm{O}_{2}\left(1 ; 1 \mathrm{~mL} \mathrm{H}_{2} \mathrm{O}_{2} 2 ; 0 \mathrm{~mL} \mathrm{H} \mathrm{H}_{2} \mathrm{O}_{2}\right)\right.$

La figura 6.1a) resume las interacciones con/sin $\mathrm{H}_{2} \mathrm{O}_{2}{ }^{*}$ volumen. Esta figura muestra la variabilidad en las concentraciones de Mn, para una relación de $\mathrm{H}_{2} \mathrm{O}_{2}$ y volumen de ácido con el que se tiene mayor concentración del analito es: 3:1 mL $\mathrm{HNO}_{3} / \mathrm{HCl}$ y con $1 \mathrm{~mL} \mathrm{H}_{2} \mathrm{O}_{2}$ con una potencia 300/600 W, con respecto con $1 \mathrm{~mL} \mathrm{H}_{2} \mathrm{O}_{2}$ y $3: 3 \mathrm{~mL}$ $\mathrm{HNO}_{3} / \mathrm{HCl}$ se obtiene concentraciones bajas para el analito.

La figura 6.2a) con interacciones rampa*con/sin $\mathrm{H}_{2} \mathrm{O}_{2}$ muestra variabilidad en las concentraciones de Mn. Para una potencia 300/600 W y con $\mathrm{H}_{2} \mathrm{O}_{2}$ se obtiene concentraciones altas para el analito con respecto de la potencia de 100/300/700 W y sin $\mathrm{H}_{2} \mathrm{O}_{2}$ donde se obtiene concentraciones bajas del analito. La figura 6.1b) con interacciones rampa*volumen muestra variabilidad en las concentraciones de Mn donde, a una potencia 300/600 W y volumen 3:1 $\mathrm{mL} \mathrm{HNO}_{3} / \mathrm{HCl}$, se obtiene mayor concentración del analito. La figura 6.2b) con interacciones volumen*rampa muestra variabilidad en las concentraciones de $\mathrm{Mn}$, donde al variar el volumen 3:1 $\mathrm{mL} \mathrm{HNO} / \mathrm{HCl}_{3}$ y potencia 300/600 $\mathrm{W}$, se obtiene concentraciones muy altas con respecto a volumen $3: 3 \mathrm{~mL} \mathrm{HNO} / \mathrm{HCl}$ y potencia 100/300/700 W. En 
este último se obtiene concentraciones muy bajas con respecto a la otra potencia. Por tanto, es óptima la primera potencia de 100/300/700 W.
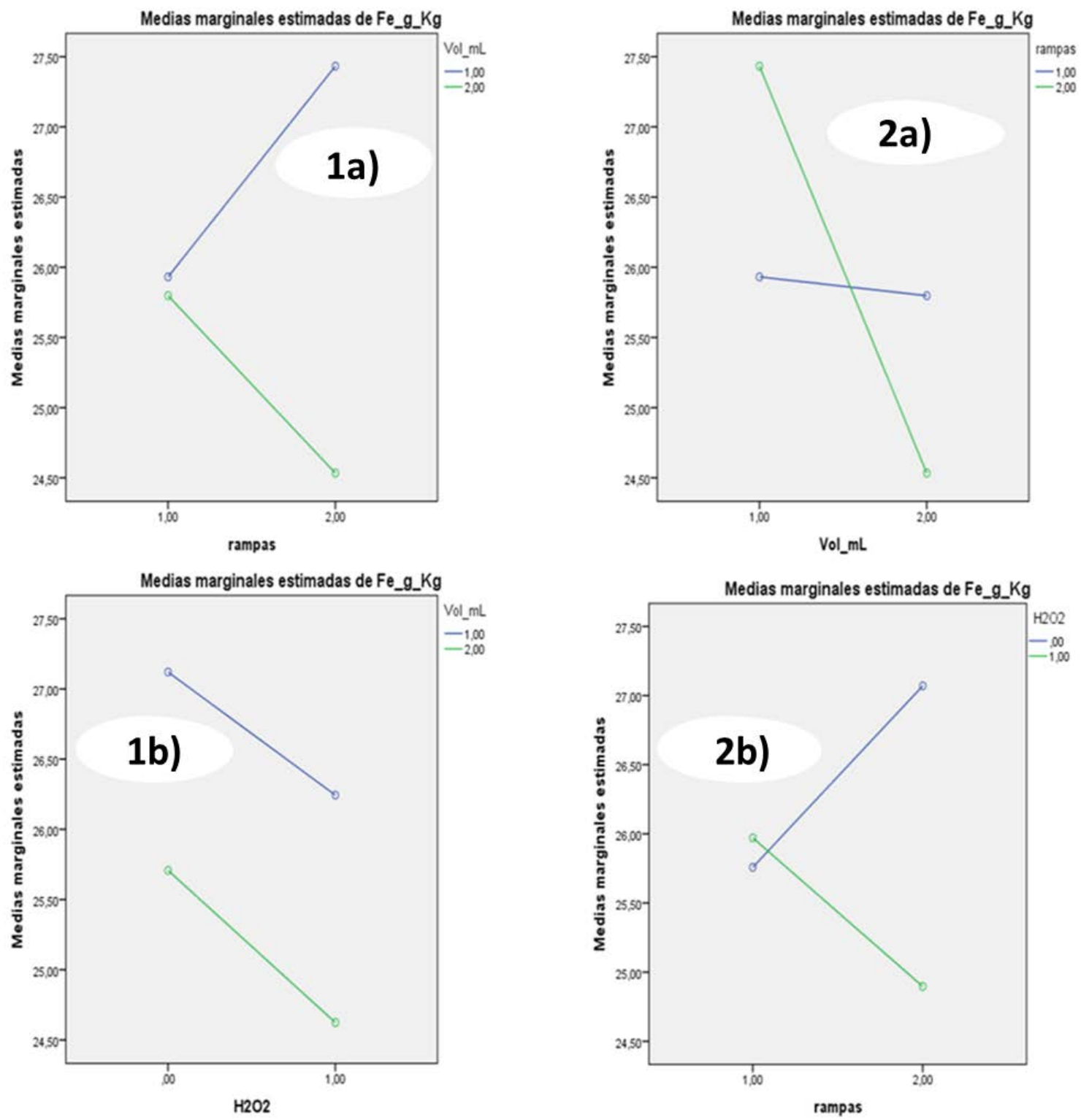

Figura 7. Diagrama factorial $2 x 2$ para el Fe variando potencia (rampa 1; 300/600 W y rampa 2; 100/300/700 W) y volumen de ácidos (1; $\mathrm{HNO}_{3}: \mathrm{HCl} 3: 1$ y 2; $\mathrm{HNO}_{3}: \mathrm{HCl} \mathrm{3:3)}$

La figura 7.1a) con interacciones rampa*volumen muestra variabilidad en las concentraciones de Fe, donde al variar los factores de potencia y volumen $\mathrm{HNO}_{3} / \mathrm{HCl}$ las concentraciones muestran diferencias significativas. Por otro lado, para potencia 100/300/700 W y $3: 3 \mathrm{~mL} \mathrm{HNO}_{3} / \mathrm{HCl}$ se obtiene concentraciones altas de Fe con respecto a potencia 300/600 W y 3:1 $\mathrm{mL} \mathrm{HNO}_{3} / \mathrm{HCl}$ que se obtiene concentraciones bajas de Fe. La figura 7.2a) con interacciones volumen*rampa muestra la variabilidad en las concentraciones de Fe, donde al variar el volumen 3:3 $\mathrm{mL} \mathrm{HNO}_{3} / \mathrm{HCl}$ y potencia $300 / 600 \mathrm{~W}$ se obtiene concentraciones relativamente bajas con respecto a volumen 3:3 $\mathrm{mL} \mathrm{HNO}_{3} / \mathrm{HCl}$ y potencia $100 / 300 / 700 \mathrm{~W}$. Además, para un volumen de $3: 1 \mathrm{~mL} \mathrm{HNO} / 3$ HCl y potencia 100/300/700 $\mathrm{W}$ se obtiene concentraciones altas con respecto a las otras condiciones. La figura 7.1b) muestra interacciones con/sin $\mathrm{H}_{2} \mathrm{O}_{2}$ *volumen con una diferencia significativa. Cuando no se añade $\mathrm{H}_{2} \mathrm{O}_{2}$ y volumen $3: 3 \mathrm{~mL}$ y $3: 1 \mathrm{~mL} \mathrm{HNO} / \mathrm{HCl}$, las concentraciones obtenidas de Fe son mayores con respecto con $\mathrm{H}_{2} \mathrm{O}_{2}$ y volumen $3: 3 \mathrm{~mL}$ y $3: 1 \mathrm{~mL} \mathrm{HNO}_{3} / \mathrm{HCl}$. La interacción volumen* con/sin $\mathrm{H}_{2} \mathrm{O}_{2}$, con mayor concentración de Fe para una potencia de 300/600 W y sin $\mathrm{H}_{2} \mathrm{O}_{2}$ y 
REVISTA BOLIVIANA DE QUÍMICA

ISSN 0250-5460 Rev. Bol. Quim. Paper edition

ISSN 2078-3949 Rev. boliv. Quim. Electronic edition

Delia M. Cori Condori et al. RBQ Vol.38, No.5, pp. 194-208, 2021

bajas concentraciones de Fe para potencias de 100/300/700 W y sin $\mathrm{H}_{2} \mathrm{O}_{2}$. La figura 7.2b) con interacciones de rampa* con/sin $\mathrm{H}_{2} \mathrm{O}_{2}$ se observa concentraciones altas con potencia 100/300/700 W y $\sin \mathrm{H}_{2} \mathrm{O}_{2}$.

Resultados de las concentraciones pseudo-totales de Zn, Mn y Fe de PK9-3 (2,0-2,7) m
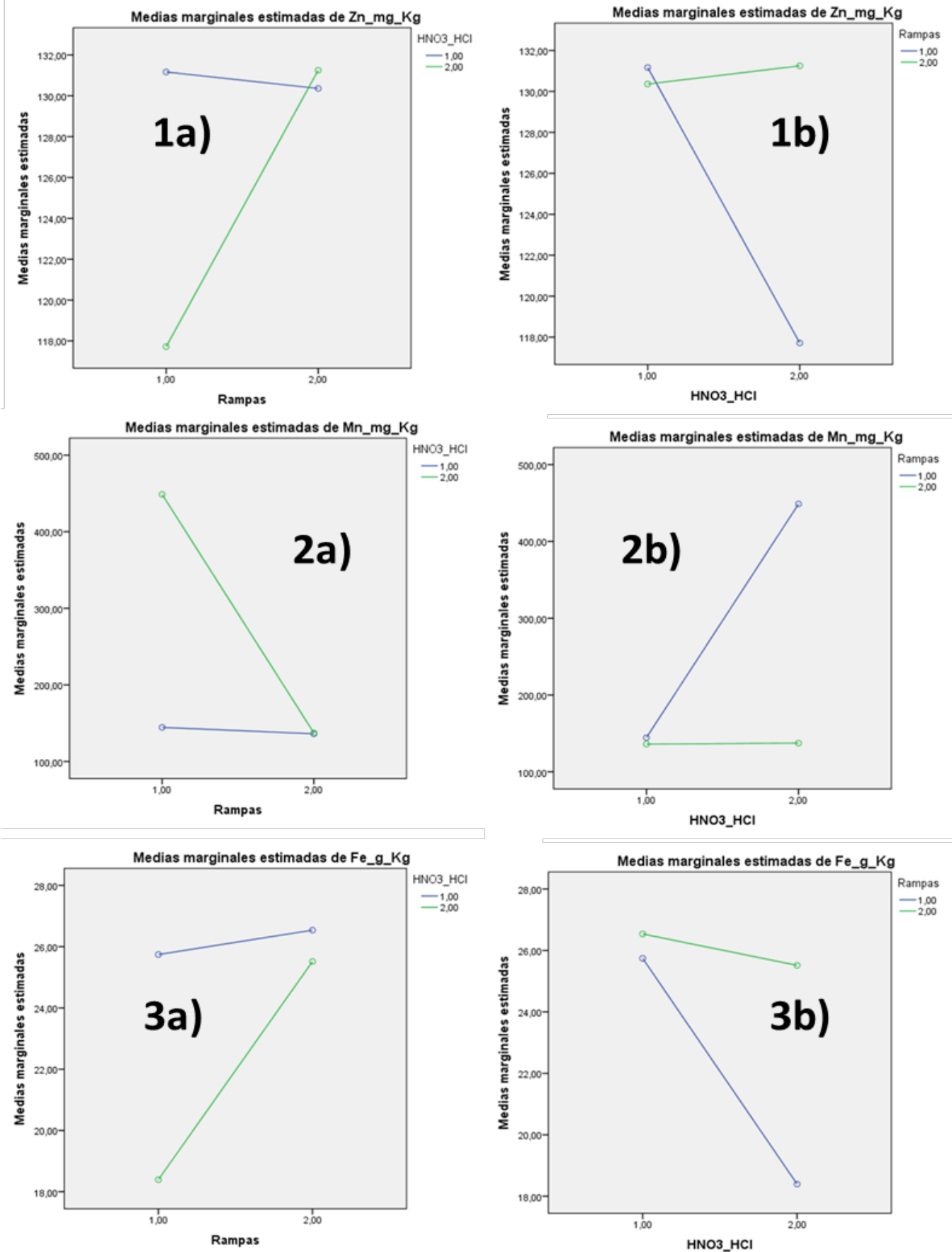

Figura 8. Diagrama factorial $2 x 2$ para el Fe, Mn y Zn variando potencia (rampa 1; 300/600 W y rampa 2; 100/300/700 W) y volumen de ácidos (1; $\left.\mathrm{HNO}_{3}: \mathrm{HCl} \mathrm{3:1} \mathrm{y} \mathrm{2;} \mathrm{HNO}_{3}: \mathrm{HCl} 3: 3\right)$

En la figura 8.1a), la interacción rampa*volumen muestra la variabilidad en las concentraciones para el Zn, variando los factores de potencia y volumen para una potencia de 100/300/700 W. Con un volumen de 3:1 $\mathrm{mL} \mathrm{HNO}_{3} / \mathrm{HCl}$ se obtiene mayor concentración de Zn. En la interacción de volumen*rampa (figura 8.1b) se observa la variabilidad en 
las concentraciones. Para una potencia de 100/300/700 W, la concentración es mayor para el Zn mientras que para una potencia 300/600 W es todo lo contrario, ya que la concentración es muy baja para el Zn. En la figura 8.2a), la interacción de la rampa*volumen para una potencia 300/600 W muestra que se tiene mayor concentración para el Mn con un volumen de 3:1 $\mathrm{mL} \mathrm{HNO}_{3}: \mathrm{HCl}$, mientras que para el volumen de 3:3 $\mathrm{mL} \mathrm{HNO}_{3}$ : $\mathrm{HCl}$ no hay interacción entre los factores de volumen y potencia, y por tanto, no hay cambio en las concentraciones. En la figura 8.2b), la interacción de volumen*rampa para una potencia 300/600 W y con un volumen 3:1 $\mathrm{mL} \mathrm{HNO}_{3}$ : $\mathrm{HCl}$ muestra que se obtiene mayor concentración para el Mn mientras que para una potencia de 100/300/700 W no presenta un cambio en las concentraciones para el Mn. La figura 8.3a) muestra la interacción rampa*volumen para el Fe, con una potencia de 100/300/700 W y con un volumen de 3:3 mL $\mathrm{HNO}_{3}$ : $\mathrm{HCl}$. En este caso, se obtiene mayor concentración de Fe. La figura 8.3b) muestra la interacción volumen*rampa para el Fe, que para una potencia de 100/300/700 W con un volumen de 3:3 $\mathrm{mL} \mathrm{HNO}_{3}$ : $\mathrm{HCl}$, se obtiene mayor concentración de Fe.

\section{CONCLUSIONES}

En conclusión, los sedimentos están conformados por arcillas con materia orgánica de color marrón y arena. El pH se clasifica como ligeramente a muy alcalino, la CE los clasifica como salino-alcalino y el \%MO clasifica de muy bajo a moderado en el contenido de MO. Por tanto, la muestra PK9-2 presenta contenidos altos en sales y \%MO debido a que está conformado principalmente por arcilla con una capa impermeable con lenta infiltración y alta evaporación.

El material de referencia MESS-3 (Marine Reference Materials for Trace Metals and other Constituents) muestra criterios de aceptación tcal < tcrit para Zn, Mn y Fe indicando que no existe diferencias significativas entre el valor experimental y el valor de referencia. Así también, la precisión determinada a través del \%CV $<5 \%$, indica que no existe variabilidad entre los datos obtenidos. Por tanto, el procedimiento óptimo es 3 horas, $\sin \mathrm{H}_{2} \mathrm{O}_{2}$ y con 3:1 $\mathrm{mL} \mathrm{HNO} / \mathrm{HCl}$. El material de referencia C17-2 (Certifícate of Analysis Metals in Soil) muestra criterios de aceptación para una potencia de 300/600 W de tcal < tcrit para Zn y tcal $\leq$ tcrit para Mn. Este caso indica que existe diferencia significativa entre el valor experimental y el valor de referencia excepto para Zn. La precisión en función al \%CV $<5 \%$ indica que no existe variabilidad en los datos obtenidos. Para potencia de 100/300/700 W el criterio de aceptación es tcal > tcrit para Zn y Mn indicando diferencia significativa entre el valor experimental y el valor de referencia. Por tanto, el procedimiento óptimo de digestión es a una potencia de 300/600 W en un tiempo de 15 minutos, sin $\mathrm{H}_{2} \mathrm{O}_{2}$ y $3: 1 \mathrm{~mL} \mathrm{HNO}_{3} / \mathrm{HCl}$.

A través de un análisis con diseño factorial 2x2 se determinó las condiciones más óptimas para determinar Fe, Mn y Zn. Por consiguiente, para la primera profundidad PK9-1 (0-0,5 m) el procedimiento óptimo es a una potencia 100/300/700 W y volumen de 3:1 $\mathrm{mL} \mathrm{HNO}_{3} / \mathrm{HCl}$ para $\mathrm{Zn}$. El procedimiento óptimo es a potencias de 300/600 W y 100/300/700 W, con volumen 3:3 $\mathrm{HNO}_{3} / \mathrm{HCl}$ para $\mathrm{Mn}$, y el procedimiento optimo es a una potencia de 300/600 W y un volumen de $3: 3 \mathrm{~mL} \mathrm{HNO}_{3} / \mathrm{HCl}$ para Fe.

Para la segunda profundidad PK9-2 (0,5-2,0 m), con contenido de \%MO las condiciones óptimas es a una potencia de 100/300/700 W, volumen 3:3 $\mathrm{mL} \mathrm{HNO}_{3} / \mathrm{HCl}$ y con $\mathrm{H}_{2} \mathrm{O}_{2}$ para $\mathrm{Zn}$, el procedimiento optimo es a una potencia 300/600 W, con/sin $\mathrm{H}_{2} \mathrm{O}_{2}$ y volumen 3:3 mL $\mathrm{HNO}_{3}$ : $\mathrm{HCl}$ para el $\mathrm{Mn}$, y las condiciones óptimas es a una potencia de 100/300/700 W y volumen 3:3 mL $\mathrm{HNO}_{3} / \mathrm{HCl}$ para Fe. Así también, para condiciones de $\sin \mathrm{H}_{2} \mathrm{O}_{2}$ con potencia 300/600 $\mathrm{W}$ y volumen $3: 3 \mathrm{~mL} \mathrm{HNO}_{3} / \mathrm{HCl}$ es óptimo para Fe.

Para la tercera profundidad PK9-3 (2,0-2,7 m) las condiciones óptimas es a un volumen 3:1 mL $\mathrm{HNO}_{3} / \mathrm{HCl}$ y potencia 100/300/700 W para Zn, las condiciones óptimas son a una potencia de 300/600 W y volumen 3:1 mL $\mathrm{HNO}_{3} / \mathrm{HCl}$ para $\mathrm{Mn}$ y las condiciones óptimas para una potencia de $100 / 300 / 700 \mathrm{~W}$ y un volumen 3:3 mL $\mathrm{HNO}_{3}$ : HCl para Fe.

Finalmente, al variar las condiciones de potencia, volumen de $\mathrm{HNO}_{3} / \mathrm{HCl}$ y con/sin $\mathrm{H}_{2} \mathrm{O}_{2}$, no se alteran considerablemente las concentraciones promedio de los ET como de $\mathrm{Zn}$ y $\mathrm{Mn}$ en las tres profundidades mostrando una $\mathrm{P}<0,05$ con excepción para el Fe donde muestra una $\mathrm{P}>0$,05. Por otro lado, las concentraciones de $\mathrm{Zn}$ que van entre 117,808 $\pm 2,391 \mathrm{mg} / \mathrm{kg}$ a $131,250 \pm 3,043 \mathrm{mg} / \mathrm{kg}$, de Mn que van entre 144,545 \pm 7,797 mg/kg a 600,156 \pm $26,108 \mathrm{mg} / \mathrm{kg}$ y Fe que van entre 18,396 $\pm 9,569 \mathrm{~g} / \mathrm{kg}$ a 28,525 \pm 4,493 g/kg varían con la profundidad de acuerdo a las características litológicas de los sedimentos.

\section{AGRADECIMIENTOS}

Al proyecto de Polución y Biorremediación Acuática financiado por la Cooperación Sueca han aportado a la investigación y al Coordinador del proyecto Lic. Jorge Quintanilla. A los revisores por los comentarios acertados para que la calidad de la publicación sea adecuada para su presentación en RBQ.

Downloadable from: Revista Boliviana de Química. http://www.bolivianchemistryjournal.org
207

Volumen 38 N5. Año 2021

http://www.scribd.com/bolivianjournalofchemistry 


\section{REFERENCIAS}

1. Lafuente, L. 2017. Optimización de un procedimiento de digestión en muestras ambientales con horno microondas y su aplicación a su separación radioquímica del PO-210, (BSc Tesis), Escuela Técnica Superior Ingenieros Industriales Valencia, Universidad Politécnica de Valencia, Valencia, España.

2. Cáceres Choque, L.F., Ramos Ramos, O.E., Choque Aspiazu, R.R. 2004. Especiación de Elementos Traza en Sedimentos del Lago Poopó, Revista Boliviana de Química, 21(1), 42 - 48.

3. Cáceres Choque, L.F., Ramos Ramos, O.E., Valdez Castro, S.N., Choque Aspiazu, R.R., Choque Mamani, R., Fernández Alcazar, S.G., Sracek, O., Bhattacharya, P. 2013. Fractionation of Heavy Metals and Assessment of Contamination of the Titicaca Lake Sediments. Environmental Monitoring and Assessment Journal, 185, 9979 - 9994. DOI: https://doi.org/10.1007/s10661-013-3306-0

4. Blanco Coarite, E. 2009. Evaluación y validación de métodos de digestión de suelos para la determinación de elementos traza, (BSc Trabajo dirigido), Universidad Mayor de San Andrés, La Paz, Bolivia, recuperado de http://repositorio.umsa.bo/xmlui/handle/123456789/17886

5. Ormachea, M., Wern, H., Johnsson, F., Bhattacharya, P., Sracek, O., Thunvik, R., Bundschuh, J. 2013. Geogenic arsenic and other trace elements in the shallow hydrogeologic system of Southern Poopó Basin, Bolivian Altiplano. Journal of Hazardous Materials, 262, 924-940. DOI: https://doi.org/10.1016/j.jhazmat.2013.06.078

6. Aruquipa, R. E. 2016. Extracción y análisis de elementos traza por Fluorescencia de Rayos X por Reflexión Total (FRXT), (BSc Tesis), Universidad Mayor de San Andrés, La Paz, Bolivia, recuperado de http://repositorio.umsa.bo/xmlui/handle/123456789/18179

7. Quino Lima, I., Ormachea Muñoz, M., Ramos Ramos, O.E., Bhattacharya, P., Quispe Choque, R., Quintanilla Aguirre, J., Sracek, O. 2019. Hydrochemical assessment with respect to arsenic and other trace elements in Lower Katari Basin, Bolivian Altiplano. Groundwater for Sustainable Development, 8, 281-293. DOI: https://doi.org/10.1016/j.gsd.2018.11.013

8. Blanco Coarite, E. 2015. Informe de consultoría Evaluación Hidrogeológica de la zona baja de la Cuenca Katari, Proyecto Polución y Biorremediación Acuática, Instituto de Investigaciones Químicas, Universidad Mayor de San Andrés, La Paz, Bolivia.

9. Quino Lima I., Ramos Ramos, O.E., Ormachea Muñoz, M., Chambi Tapia, M.I., Quintanilla Aguirre J., Arslan Ahmad, Jyoti Prakash Maity, Md. Tahmidul Islam, Prosun Bhattacharya. 2021. Geochemical mechanisms of natural arsenic mobility in the hydrogeologic system of Lower Katari Basin, Bolivian Altiplano. Journal of Hydrology, Volume 594, March 2021, 125778.

10. Passos, E. A., Alves, J. C., Santos, I. S., Alves, J. P., Garcia, C. A., \& Costa, A. C. 2010. Assessment of trace metals contamination in estuarine sediments using asequential extraction technique and principal component analysis. Microchem J. 96, 50-57.

11. McKean J. S. 1993. Manual de análisis de suelos y tejido vegetal. Centro Internacional de Agricultura Tropical·CIAT. Documento de trabajo Nro. 129.

Downloadable from: Revista Boliviana de Química. http://www.bolivianchemistryjournal.org
208

Volumen 38 Nº5. Año 2021

http://www.scribd.com/bolivianjournalofchemistry 